\title{
COMBINING MONTE CARLO SIMULATION WITH HEURISTICS FOR SOLVING THE INVENTORY ROUTING PROBLEM WITH STOCHASTIC DEMANDS
}

\author{
José Cáceres-Cruz \\ Angel A. Juan \\ IN3-Computer Science Dept. \\ Open University of Catalonia \\ Barcelona, 08018, Spain \\ Tolga Bektas \\ School of Management \\ University of Southampton \\ Southampton, SO17 1BJ, UK
}

\author{
Scott E. Grasman \\ Industrial and Systems Engineering Dept. \\ Rochester Institute of Technology \\ Rochester, NY 14623-5603, USA \\ Javier Faulin \\ Dept. Statistics and Operations Research \\ Public University of Navarre \\ Pamplona, 31006, Spain
}

\begin{abstract}
In this paper, we introduce a simulation-based algorithm for solving the single-period Inventory Routing Problem (IRP) with stochastic demands. Our approach, which combines simulation with heuristics, considers different potential inventory policies for each customer, computes their associated inventory costs according to the expected demand in the period, and then estimates the marginal routing savings associated with each customer-policy entity. That way, for each customer it is possible to rank each inventory policy by estimating its total costs, i.e., both inventory and routing costs. Finally, a multi-start process is used to iteratively construct a set of promising solutions for the IRP. At each iteration of this multi-start process, a new set of policies is selected by performing an asymmetric randomization on the list of policy ranks. Some numerical experiments illustrate the potential of our approach.
\end{abstract}

\section{INTRODUCTION}

Today, one of the most important concepts in supply chain management is that of replacing sequential decision making with global decision making, where all parties in the supply chain determine the best policy for the entire system. Inventory and transportation systems are good examples of sequential decision making. However, driven by business practices such as vendor managed inventory (VMI), integrated inventory and transportation systems have received much recent attention (Kleywegt et al. 2004). VMI is a supply chain centralized control initiative where the supplier is authorized to manage inventories of the retailers and to make decisions such as when and how much inventory to ship to the retailer. VMI is seen as an effective means of managing inventory through the strategic use of technologies which enable the flow of information throughout the entire supply chain. Despite the potential benefits, and probably due to its complexity, only a relatively small number of articles have analytically approached the issue of integrating decisions. This issue is known in the literature as the Inventory Routing Problem or IRP (Campbell et al. 2002). Therefore, model formulations with exact or approximate solution procedures are still needed to assist with the widespread adoption of VMI and use of synchronized inventory and transportation systems.

In this paper, a hybrid approach is proposed. Our approach combines Monte Carlo simulation (MCS) with a multi-start asymmetric randomization of a classical routing heuristic. We consider a single-period 


\section{Caceres-Cruz, Juan, Grasman, Bektas, and Faulin}

IRP consisting of multiple retailers with stochastic demands and a single distribution depot. Since final demands at the retailer centers are assumed to be random variables, potential stock-outs are considered in our model. In the centralized version of this problem that we are addressing, no assumption is made about the inventory policy at an individual retailer. The distribution depot will analyze the inventory position of the retailers and make joint inventory and routing decisions that minimize the total cost to the system, which consists of treated individually retailers.

Another aspect to notice is that most of the existing literature has considered the IRP as a long-term, multi-period problem (Campbell and Savelsbergh 2002). This is especially the case when the final demands at the retailer centers are assumed to be deterministic. However, we feel that it is also important to study the singleperiod problem, particularly in those scenarios characterized by: (a) information and communication tools that are able to efficiently monitor and report retailers' stock levels at the end of each period; and (b) random demands with a high variability, which makes it difficult to forecast future inventory levels.

The remainder of the article is structured as follows: Section 2 briefly describes the IRP with stochastic demands and reviews some related work; Section 3 gives an overview explanation of our approach; Section 4 presents and discusses some numerical experiments that serve to both illustrate and validate our approach; and finally, Section 5 summarizes the main contributions and results of this work.

\section{THE IRP WITH STOCHASTIC DEMANDS AND RELATED WORK}

The IRP with stochastic demands, which is an NP-hard problem, can be described as follows: consider a Capacitated Vehicle Routing Problem (CVRP) with $n$ nodes or retailing centers (RC), plus the depot. Each RC owns an inventory, which is managed by the central depot. For each RC, the inventory level at the end of a period depends on the initial stock level and also on the end-clients' demands during that period. These end-clients' demands are stochastic in nature. In our approach, we will assume that, for each $\mathrm{RC}$, it has been possible to use historical data to model end-clients' demands through a theoretical or empirical probability distribution. Notice that no particular assumption is made on the type of distribution used to model these demands. Therefore, at the end of each period there might be some costs associated with inventory holding and inventory stock-outs. These costs might be incorporated into the decisionmaking process and integrated with (added to) the distribution or routing costs, which are usually based on traveling distances and/or times. At the end of each period, inventory levels are registered by the RC and updated in the central depot, so that a new routing strategy is defined for the new period taking into account the new data. Our goal is to minimize total expected costs (distribution plus inventory-related costs) in each single-period scenario.

Some of the first work on the IRP with stochastic demands is due to Federgruen and Zipkin (1984). They address the single-period combined problem of "allocating a scarce resource available at some central depot among several locations, each experiencing a random demand pattern". They propose a mathematical model and design a modified interchange heuristic as well as an exact algorithm to solve some randomly generated instances with up to 75 nodes. In Bard et al. (1998), the authors study the IRP with satellite facilities (depots geographically scattered throughout the service area). Interestingly, the authors use a randomized version of the classical Clarke and Wright Savings (CWS) heuristic (Clarke and Wright 1964) to solve routing instances with up to 500 nodes in about two hours. They show that this randomized heuristic outperforms other algorithms, including a Greedy Randomized Adaptive Search Procedure (GRASP). The GRASP procedure consists in successive constructions of a greedy randomized solution and subsequent iterative improvements of it using local search procedures (Feo and Resende 1989, 1995). While the randomness process they propose is based on a uniform (symmetric) distribution, in this paper we make use of an asymmetric distribution. Berman and Larson (2001) focus on the problem associated with the distribution of industrial gases to replenish customer tanks with random demands. They propose four dynamic-programming algorithms for solving the associated problem. In Jarugumilli et al. (2006), the authors make use of a modified version of the $\mathrm{A}^{*}$ algorithm (originally used in path-finding in graphs) to solve the stochastic IRP with a single vehicle. Hvattum et al. (2009) address the stochastic IRP with an infinite horizon as a Markov process. They formulate a scenario tree in order to examine a finite horizon 


\section{Caceres-Cruz, Juan, Grasman, Bektas, and Faulin}

as a good approximation to the infinite horizon model. Again, since solving stochastic IRP cases with a Markov process is impractical for all but the smallest instances, they proposed to employ a GRASP heuristic. Finally, Bertazzi et al. (2011) undertake a stochastic IRP with stock-outs and a finite horizon. They assume an order-to-level policy i.e., "the quantity sent to each retailer is such that its inventory level reaches the maximum level". They present a dynamic programming model and propose a hybrid rollout algorithm. The authors use a randomly generated set of instances with up to 50 nodes and 6 periods.

The approach we present in this paper is similar to with some of the previous work, especially with those considering stochastic demands, stock-outs, and rollout periods. However, our approach has some significant differences: (a) we consider several replenishment policies; (b) we use a hybrid algorithm combining simulation with a metaheuristic, which allows us to obtain 'good' solutions to large-size instances in a reasonable time; (c) we promote the use of asymmetric randomization of heuristics; and (d) we propose a completely described set of instances (not a randomly generated one).

\section{AN OVERVIEW OF OUR APPROACH}

Our approach focuses on solving the single-period IRP with stochastic demands and possible stock-outs. In this context, we propose a hybrid approach which combines MCS with an efficient CVRP heuristic. MCS can be defined as a set of techniques that make use of random numbers and statistical distributions to solve certain stochastic and deterministic problems (Law 2007). When properly combined with heuristic techniques, MCS has proved to be extremely useful for solving stochastic VRPs (Juan et al. 2011a). Our approach is also based on the SR-GCWS-CS randomized algorithm proposed by Juan et al. (2011b) for solving the CVRP. This algorithm makes use of a pseudo-geometric distribution to induce an asymmetric randomization process into the CWS heuristic; and it also employs a memory-based local search.

In this paper we consider five different service policies or inventory levels for each customer. Specifically, given a customer, we consider the following policies: (a) no refill for that customer; (b) refill up to one quarter of its capacity; (c) refill up to half of its capacity; (d) refill up to three quarters of its capacity; and (e) full refill. The flow diagram of our algorithm is depicted in Figure 1 and described next as a fivestep procedure. In fact, our methodology could consider more intermediate policies if necessary, which makes it quite flexible. Of course, considering more intermediate policies can lead to slightly better solutions, but will also increase somewhat the computational effort. Thus, for each combination of customerservice policy, MCS is used to obtain estimates of the inventory costs associated with it - "including both surplus and shortage costs". As with any other approximate approach, this method is unlikely to produce an optimal solution, but it can produce near-optimal solutions in a reasonable amount of time.

First, for each customer in the problem, the expected inventory costs associated with each eligible policy are estimated throughout by MCS. Here, both potential surpluses and shortages (stock-outs) are considered for each of the refill policies described in the previous section. In the second step of the procedure, we consider the worst-case scenario from a distribution point of view, i.e., all customers receive a full refill. In this scenario, a fast heuristic is used to obtain a 'good' solution for the associated CVRP. This solution will provide an estimate of the total distribution costs under the full-refill policy. In the third step, we estimate for each customer the routing "marginal savings", i.e., the reduction in distribution costs associated with each non-full-refill policy. In order to do this, a fast heuristic is used to solve a large set of CVRPs. A fast heuristic should be employed here since this step requires us to solve one CVRP for each customer-policy combination, i.e., for each customer and for each non-full-refill policy. Once these marginal costs have been estimated, for each customer an approximated value for the total costs associated with each eligible policy can be obtained by simply adding up estimated routing and inventory costs. Thus, for each customer, the associated eligible policies can be sorted from lower to higher total costs, thus defining a priority policy rank for each customer. In the fourth step, the 'top' policy for each customer (i.e., the one showing the lowest total cost) is selected, and a pseudo-optimal solution is obtained for the corresponding CVRP by using an efficient algorithm, e.g., the SR-GCWS-CS (Juan et al. 2011b). Finally, in the fifth step, a multi-start process is carried out. At each iteration of this multi-start process, a new policy is randomly selected for each customer and, in a similar way as in the previous step, a new 
pseudo-optimal solution is obtained for the corresponding CVRP. The best solution found so far is recorded. Notice, however, that the random selection process uses an asymmetric distribution (like the geometric). With this, we aim at assigning more probabilities of being selected to those policies which are located at the top positions of each customer's ranking of policies. Using a multi-start approach makes it difficult for the algorithm to get trapped in a local minimum.

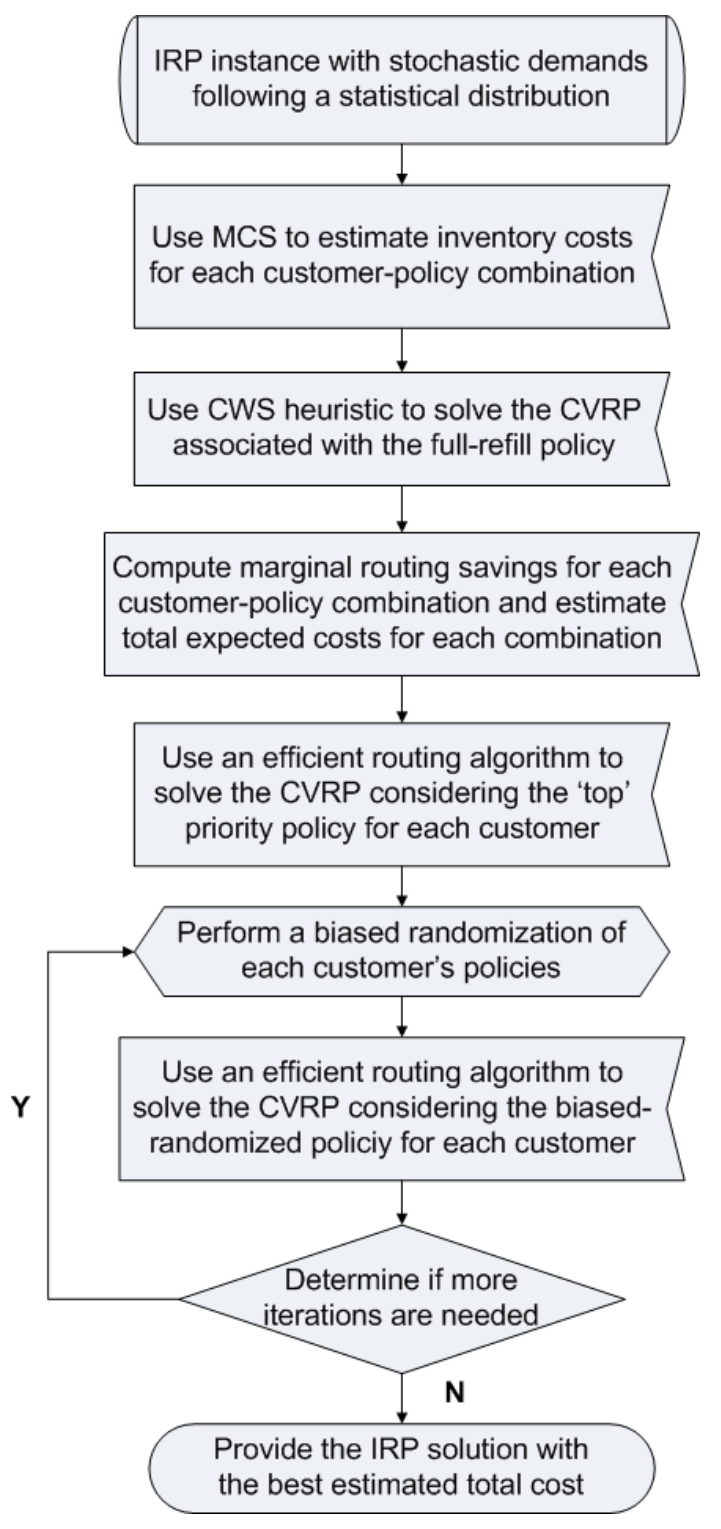

Figure 1. Flow diagram for our algorithm.

\section{NUMERICAL EXPERIMENTS}

In the CVRP literature, there exists a classical set of well-known benchmarks commonly used to test new CVRP algorithms. However, this is not the case for the single-period IRP with stochastic demands and stock-outs. For this reason, and with the goal of providing complete information about the set of benchmarks employed so that other researchers can use them, we have developed our own set of data by generalizing the well-known datasets $A$ and $B$ from the CVRP literature (Augerat et al. 1995). These datasets 


\section{Caceres-Cruz, Juan, Grasman, Bektas, and Faulin}

consist of 27 small- and medium-size test instances (available at http://www.branchandcut.org/VRP). A natural generalization of these datasets has been carried out by using random demands instead of deterministic ones. So, for each instance, while we decided to keep all node coordinates and vehicle capacities, we changed $d_{i}$, the deterministic demand of customer $i$ (for all $i=1,2, \ldots, n$ ) to the probabilistic demand $D_{i}$ with $E\left[D_{i}\right]=d_{i}$. Since we use MCS, these random demands can follow any probability distribution having a finite mean. For the numerical experiments of this paper, however, we will assume that $D_{i}$ follows a $\operatorname{LogNormal}$ distribution with $E\left[D_{i}\right]=d_{i}$ and $\operatorname{Var}\left[D_{i}\right]=0.25 d_{i}$. Other classical hypotheses design the demand random variables as following a Normal distribution, but this assumption is not realistic due to the fact that the Normal variable can take negative values which are not allowed to describe a demand. Regarding the inventory part of the problem, we will also make the following assumptions:

a) For each customer $i$, its maximum inventory capacity is defined as $\max _{i}=2 d_{i}$. As it usually happens in real-life, customers with higher expected demands will have higher inventory capacities.

b) In correspondence with the distribution policies considered, the quantity that can be delivered to each customer, $q_{i}$, can only take a discrete number of values, i.e., according to the policies described above, $q_{i}$ can only take floating values in the set $\left\{0,0.5 d_{i}, d_{i}, 1.5 d_{i}, 2 d_{i}\right\}$.

c) Trying to imitate a realistic scenario, in which it is likely that different customers will present different starting stock levels, the initial inventory level at customer $i, L_{i}$, is assigned according to the following expression:

$$
L_{i}=\left\{\begin{array}{cc}
0 & \text { if } i \text { is odd and a multiple of } 3 \\
0.5 d_{i} & \text { if } i \text { is odd and not a multiple of } 3 \\
d_{i} & \text { if } i \text { is even and a multiple of } 4 \\
1.5 d_{i} & \text { if } i \text { is even and not a multiple of } 4
\end{array}\right.
$$

Finally, regarding the inventory costs, these must be of the same order of magnitude as the routing costs in order to reach a proper balance between those two costs. In fact, this helps to make sense of not serving some customers under certain conditions, e.g., high inventory levels and low stock-out costs. In order to attain this goal, we have used the following expression to define the inventory costs, $I C_{i}$, associated with each customer:

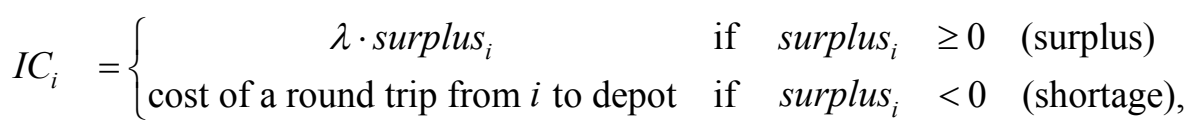

where surplus $_{i}=L_{i}+q_{i}-D_{i}$. Notice that $\lambda$ represents the cost per unit of stock at the end of the period. In the numerical experiments, we have used a value of $\lambda=0.01$. This value was chosen in order to balance routing and inventory costs in such a way that it might be not worthwhile to serve some of the customers. Thus those nodes with a low probability of suffering a stock-out, or those with low penalty costs in case they suffer from a stock-out are being adequately considered in our approach.

Our algorithm was implemented as a Java application and used to run the 27 instances described above on an Intel Xeon E5603 at $1.60 \mathrm{Ghz}$ and $8 \mathrm{~GB}$ of RAM. For each instance, a single run with a total maximum time of 3 minutes was employed. Table 1 shows the results obtained in our experiments for the following policies:

(a) Full-refill policy, i.e., all customers are served up to their maximum capacity.

(b) Top policy, i.e., each customer is served according to the 'best' policy in its sorted priority list of policies (notice that this top policy could imply that the customer does not need to be served).

(c) Asymmetrically-randomized policy, i.e., as explained in our approach, each customer is served according to a policy which has been asymmetric-randomly selected from its sorted policies list.

Additionally, Table 1 also shows the percentage gaps between the solution obtained using each policy 


\section{Caceres-Cruz, Juan, Grasman, Bektas, and Faulin}

and our best solution, i.e., the one obtained with the asymmetric-randomized process. Positive gaps imply that the total cost obtained with the asymmetric-randomized process is lower (and therefore better) than the total cost obtained with the alternative method.

Table 1: Results for full-refill, top, and asymmetrically-randomized policies $(\lambda=0.01$, max. computation time $=3$ minutes).

\begin{tabular}{|c|c|c|c|c|c|c|c|c|c|c|c|}
\hline \multirow[b]{2}{*}{ Instance } & \multicolumn{3}{|c|}{ FULL-REFILL POLICY (1) } & \multicolumn{3}{|c|}{ TOP POLICY (2) } & \multicolumn{3}{|c|}{$\begin{array}{c}\text { ASYMMETRICALLY- } \\
\text { RANDOMIZED POLICY (3) }\end{array}$} & \multicolumn{2}{|c|}{ GAPS } \\
\hline & $\begin{array}{c}\text { Routing } \\
\text { Costs }\end{array}$ & $\begin{array}{c}\text { Inventory } \\
\text { Costs }\end{array}$ & $\begin{array}{l}\text { Total } \\
\text { Costs } \\
\end{array}$ & $\begin{array}{l}\text { Routing } \\
\text { Costs }\end{array}$ & $\begin{array}{l}\text { Inventory } \\
\text { Costs }\end{array}$ & $\begin{array}{l}\text { Total } \\
\text { Costs } \\
\end{array}$ & $\begin{array}{c}\text { Routing } \\
\text { Costs }\end{array}$ & $\begin{array}{c}\text { Inventory } \\
\text { Costs }\end{array}$ & $\begin{array}{l}\text { Total } \\
\text { Costs } \\
\end{array}$ & (1)-(3) & (2)-(3) \\
\hline A-n32-k5 & 981.38 & 4.72 & 986.10 & 600.08 & 24.12 & 624.21 & 547.49 & 50.66 & 598.15 & $64.86 \%$ & $4.36 \%$ \\
\hline A-n33-k5 & 805.34 & 4.62 & 809.96 & 421.17 & 41.70 & 462.87 & 421.17 & 41.70 & 462.87 & $74.99 \%$ & $0.00 \%$ \\
\hline A-n33-k6 & 841.43 & 5.72 & 847.15 & 504.88 & 27.20 & 532.08 & 503.92 & 23.49 & 527.41 & $60.62 \%$ & $0.88 \%$ \\
\hline A-n37-k5 & 751.56 & 5.66 & 757.22 & 437.12 & 26.53 & 463.65 & 427.67 & 32.49 & 460.15 & $64.56 \%$ & $0.76 \%$ \\
\hline A-n38-k5 & 797.92 & 5.53 & 803.45 & 529.83 & 18.60 & 548.44 & 529.83 & 18.60 & 548.44 & $46.50 \%$ & $0.00 \%$ \\
\hline A-n39-k6 & 940.61 & 5.74 & 946.35 & 491.57 & 47.61 & 539.18 & 491.57 & 47.61 & 539.18 & $75.52 \%$ & $0.00 \%$ \\
\hline A-n45-k6 & $1,102.03$ & 6.15 & $1,108.18$ & 628.52 & 33.07 & 661.60 & 628.52 & 33.07 & 661.60 & $67.50 \%$ & $0.00 \%$ \\
\hline A-n45-k7 & $1,423.45$ & 7.15 & $1,430.60$ & 683.32 & 52.95 & 736.27 & 683.32 & 52.95 & 736.27 & $94.30 \%$ & $0.00 \%$ \\
\hline A-n55-k9 & $1,372.01$ & 8.66 & $1,380.67$ & 688.91 & 61.03 & 749.94 & 688.91 & 61.03 & 749.94 & $84.10 \%$ & $0.00 \%$ \\
\hline A-n60-k9 & $1,683.88$ & 8.90 & $1,692.78$ & 765.50 & 78.11 & 843.60 & 765.50 & 78.11 & 843.60 & $100.66 \%$ & $0.00 \%$ \\
\hline A-n61-k9 & $1,146.35$ & 9.15 & $1,155.50$ & 559.50 & 51.32 & 610.82 & 559.50 & 51.32 & 610.82 & $89.17 \%$ & $0.00 \%$ \\
\hline A-n63-k9 & $1,941.07$ & 9.32 & $1,950.40$ & 952.33 & 72.16 & $1,024.49$ & 952.33 & 72.16 & $1,024.49$ & $90.38 \%$ & $0.00 \%$ \\
\hline A-n65-k9 & $1,372.32$ & 9.83 & $1,382.16$ & 672.13 & 62.72 & 734.86 & 672.13 & 62.72 & 734.86 & $88.09 \%$ & $0.00 \%$ \\
\hline A-n80-k10 & $2,153.31$ & 10.65 & $2,163.96$ & $1,019.82$ & 148.77 & $1,168.59$ & $1,019.82$ & 148.77 & $1,168.59$ & $85.18 \%$ & $0.00 \%$ \\
\hline B-n31-k5 & 807.06 & 4.27 & 811.33 & 495.18 & 12.66 & 507.84 & 453.40 & 34.50 & 487.89 & $66.29 \%$ & $4.09 \%$ \\
\hline B-n35-k5 & $1,179.77$ & 5.49 & $1,185.27$ & 713.53 & 18.87 & 732.40 & 532.69 & 48.76 & 581.45 & $103.85 \%$ & $25.96 \%$ \\
\hline B-n39-k5 & 652.67 & 6.05 & 658.72 & 357.73 & 18.35 & 376.08 & 357.73 & 18.35 & 376.08 & $75.15 \%$ & $0.00 \%$ \\
\hline B-n41-k6 & 931.99 & 5.87 & 937.86 & 536.08 & 24.35 & 560.43 & 510.57 & 35.99 & 546.56 & $71.59 \%$ & $2.54 \%$ \\
\hline B-n45-k5 & 866.29 & 6.55 & 872.84 & 432.14 & 32.88 & 465.03 & 432.14 & 32.88 & 465.03 & $87.70 \%$ & $0.00 \%$ \\
\hline B-n50-k7 & 891.43 & 6.34 & 897.76 & 480.60 & 33.68 & 514.28 & 480.60 & 33.68 & 514.28 & $74.57 \%$ & $0.00 \%$ \\
\hline B-n52-k7 & 926.11 & 7.06 & 933.17 & 458.30 & 45.18 & 503.48 & 458.30 & 45.18 & 503.48 & $85.34 \%$ & $0.00 \%$ \\
\hline B-n56-k7 & 761.51 & 7.09 & 768.60 & 434.08 & 20.74 & 454.82 & 434.08 & 20.74 & 454.82 & $68.99 \%$ & $0.00 \%$ \\
\hline B-n57-k9 & $1,940.14$ & 8.36 & $1,948.50$ & $1,136.54$ & 39.62 & $1,176.17$ & $1,066.85$ & 84.92 & $1,151.77$ & $69.18 \%$ & $2.12 \%$ \\
\hline B-n64-k9 & $1,070.40$ & 9.54 & $1,079.94$ & 604.24 & 31.42 & 635.66 & 604.24 & 31.42 & 635.66 & $69.89 \%$ & $0.00 \%$ \\
\hline B-n67-k10 & $1,311.06$ & 10.09 & $1,321.16$ & 701.14 & 52.95 & 754.09 & 701.14 & 52.95 & 754.09 & $75.20 \%$ & $0.00 \%$ \\
\hline B-n68-k9 & $1,541.41$ & 9.59 & $1,551.00$ & 735.64 & 67.35 & 803.00 & 735.64 & 67.35 & 803.00 & $93.15 \%$ & $0.00 \%$ \\
\hline B-n78-k10 & $1,423.15$ & 9.99 & $1,433.14$ & 764.51 & 56.54 & 821.05 & 764.51 & 56.54 & 821.05 & $74.55 \%$ & $0.00 \%$ \\
\hline Averages & $1,170.95$ & 7.34 & $1,178.29$ & 622.39 & 44.46 & 666.85 & 608.28 & 49.55 & 657.83 & $79.12 \%$ & $1.37 \%$ \\
\hline
\end{tabular}

From Table 1, it is clear that the best results are obtained using our asymmetric-randomization approach (average gap of almost $80 \%$ with respect to the full refill policy), i.e., using a different refill strategy for each node according to different factors such as distance from the depot, current inventory level, expected demand, demand variability, etc. Notice also that using the top strategy for each node as proposed in an intermediate stage of our approach provides a competitive solution for most instances (average gap of $1.37 \%$ with respect to our best solution). However, using non-personalized refill strategies - "i.e., using the same refill strategy for all the customers as proposed in most existing articles" - is not a good methodology since it results in considerably higher costs.

Finally, Figure 2 illustrates two different routing solutions obtained with the two different refill policies proposed in our algorithm ('top' and 'asymmetric-randomization') for the A-n32-k5 instance. Squares (₫) represent customers receiving a full-refill. Diamonds ( $\downarrow$ ) show customers receiving a $3 / 4-$ refill. Triangles $(\boldsymbol{\Delta})$ represent customers receiving a $1 / 2$-refill. Circles $(\bullet)$ represent customers receiving a 
1/4-refill. Finally, stars (*) represent non-served customers. On the left side, the top policy solution proposes to visit some nodes with a specific inventory refill strategies and a routing configuration. While, on the right side, the asymmetrically-randomized policy applies some other inventory policies to some nodes. Thus the subset of served nodes are distinct. Both solutions propose three routes but the compositions of each is different thanks to the application of dissimilar refill policies to each node. The asymmetrically-randomized policy allows to find a better and balanced configurations with individual policies for each node.
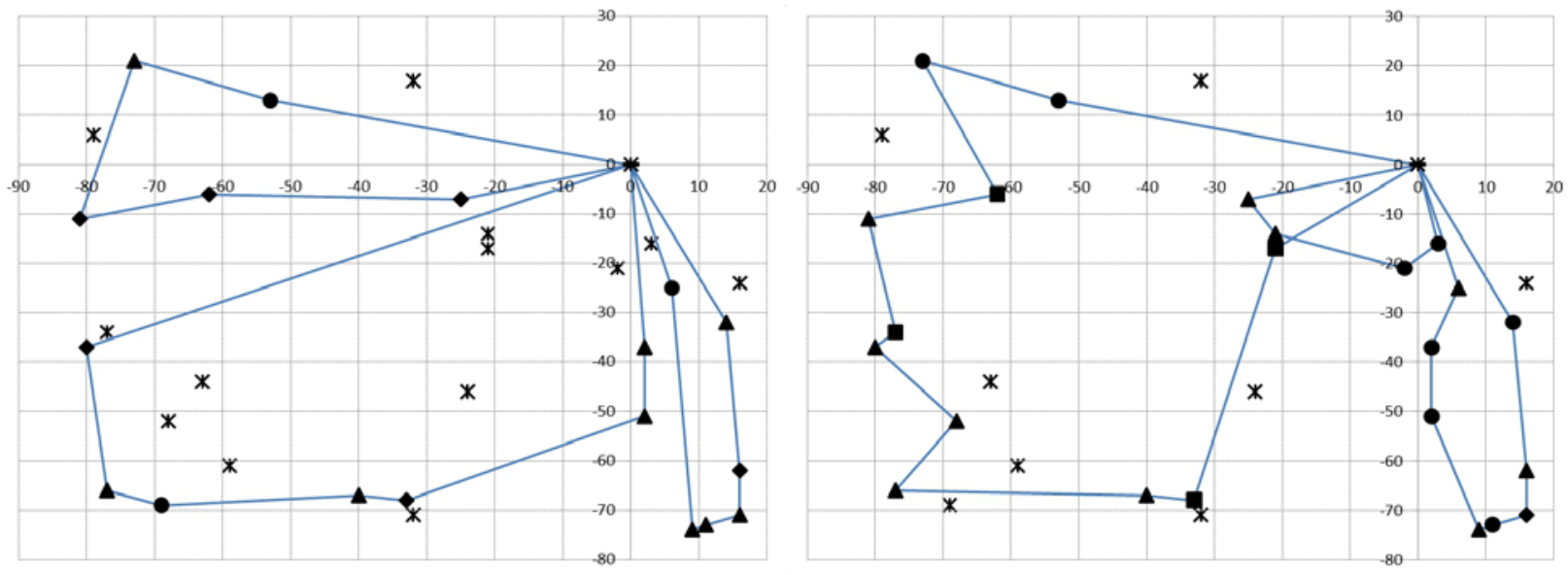

Figure 2: Solutions obtained for A-n32-k5 using the 'top' refill and 'asymmetrically-randomized' refill

policies.

\section{CONCLUSIONS}

In this paper, we have presented a hybrid approach for solving the Inventory Routing Problem with Stochastic Demands (IRPSD) and with stock-outs. The IRPSD is a challenging research area because it introduces random behavior into a problem combining two steps of supply chain management -"inventory control and distribution planning". The proposed approach integrates Monte Carlo simulation into different key phases of a heuristic approach. One of the main contributions of our methodology is that it can consider personalized refill policies for each customer, which contributes to significantly to reduce total costs over other approaches using standard refill policies. A set of benchmarks for the IRPSD were developed and a realistic expression to model inventory costs was also proposed. A complete set of tests have been performed to illustrate the methodology and analyze its efficiency as well as its potential benefits.

\section{ACKNOWLEDGMENTS}

This work has been partially supported by the Spanish Ministry of Science and Innovation (TRA201021644-C03) and by the Ibero-American Programme for Science, Technology and Development (CYTED2010-511RT0419) in the context of the IN3-HAROSA (http://dpcs.uoc.edu) and Sustainable TransMET (Government of Navarre, Spain) research networks. 


\section{REFERENCES}

Augerat, P., J.M. Belenguer, E. Benavent, A. Corberán, D. Naddef, and G. Rinaldi. 1995. "Computational Results with a Branch and Cut Code for the Capacitated Vehicle Routing Problem". Research Report 949-M, Université Joseph Fourier, Grenoble, France.

Bard, J. F., L. Huang, P. Jaillet, and M. Dror. 1998. "A Decomposition Approach to the Inventory Routing Problem with Satellite Facilities”. Transportation Science 32:189-203.

Berman, O., and R. C. Larson. 2001. "Deliveries in an Inventory/Routing Problem Using Stochastic Dynamic Programming”. Transportation Science 35(2):192-213.

Bertazzi, L, A. Bosco, F. Guerriero, and D. Lagana. 2011. "A Stochastic Inventory Routing Problem with Stock-out". Transportation Research Part C, (in press) doi: 10.1016/j.trc.2011.06.003.

Campbell, A., L. W. Clarke, and M. Savelsbergh. 2002. "Inventory Routing in Practice”. In The Vehicle Routing Problem, SIAM Monographs on Discrete Mathematics and Applications, Edited by P. Toth and D. Vigo. Philadelphia.

Clarke, G., and J. W. Wright. 1964. "Scheduling of Vehicles from a Central Depot to a Number of Delivery points". Operations Research 12:568-581.

Federgruen, A., and P. Zipkin. 1984. “A Combined Vehicle Routing and Inventory Allocation Problem”. Operations Research 32:1019-1036.

Feo, T.A. and Resende, M.G.C. 1989. "A probabilistic heuristic for a computationally difficult set covering problem". Operations Research Letters, 8:67-71.

Feo, T.A. and Resende, M.G.C. 1995. "Greedy randomized adaptive search procedures". Journal of Global Optimization, 6:109-133, 1995.

Golden, B., S. Raghavan, and E. Wasil. 2008. The Vehicle Routing Problem: Latest Advances and New Challenges. Springer, New York.

Hvattum, L. M., A. Løkketangen, and G. Laporte. 2009. "Scenario Tree Based Heuristics for Stochastic Inventory Routing Problems”. INFORMS Journal on Computing 21(2):268-285.

Jarugumilli, S., S.E. Grasman, and S. Ramakrishnan. 2006. "A Simulation Framework for Real-Time Management and Control of Inventory Routing Decisions". In Proceedings of the 2006 Winter Simulation Conference. Edited by L. F. Perrone, F. P. Wieland, J. Liu, B. G. Lawson, D. M. Nicol, and R. M. Fujimoto, 1485-1492. Piscataway, New Jersey: Institute of Electrical and Electronics Engineers, Inc.

Juan, A., J. Faulin, S. Grasman, D. Riera, J. Marull, and C. Mendez. 2011a. "Using Safety Stocks and Simulation to Solve the Vehicle Routing Problem with Stochastic Demands". Transportation Research Part C 19:751-765.

Juan, A., J. Faulin, J. Jorba, D. Riera, D. Masip, and B. Barrios, 2011b. "On the Use of Monte Carlo Simulation, Cache and Splitting Techniques to Improve the Clarke and Wright Savings Heuristics". Journal of the Operational Research Society, 62(6):1085-1097.

Kleywegt, A. J., V. S. Nori, and M. W. Savelsbergh. 2004. "Dynamic Programming Approximations for a Stochastic Inventory Routing Problem”. Transportation Science 38(1):42-70.

Law, A. 2007. Simulation Modeling and Analysis. $4^{\text {th }}$ edition. McGraw-Hill: Boston.

\section{AUTHOR BIOGRAPHIES}

JOSE CACERES-CRUZ is a Ph.D. student of the Information and Knowledge Society Doctoral Programme in the Open University of Catalonia. He holds a Specialization on Information Systems from the Central University of Venezuela and a Computer Engineering degree from the Simón Bolívar University. His main research interests are related to optimization of vehicle routing on realistic scenarios, and simulation solutions. His email address is jcaceresc@uoc.edu. 
ANGEL A. JUAN is an Associate Professor of Applied Optimization and Simulation in the Computer Science Department at the IN3-Open University of Catalonia (Barcelona, Spain). Dr. Juan holds a Ph.D. in Applied Computational Mathematics, an M.S. in Information Systems and Technology, and an M.S. in Applied Mathematics. His research interests include Applied Optimization and Randomized Algorithms. He has published over 100 peer-reviewed papers in international journals, books, and proceedings. Currently, he is the coordinator of the CYTED-IN3-HAROSA@IB Network and an editorial board member of both the Int. J. of Data Analysis Techniques and Strategies and the Int. J. of Information Systems and Social Change. His e-mail is ajuanp@gmail.com and his web page is http://ajuanp.wordpress.com.

SCOTT GRASMAN is a Full Professor and Department Head of Industrial and Systems Engineering at Rochester Institute of Technology. Dr. Grasman received his B.S., M.S., and Ph.D. degrees in Industrial and Operations Engineering from the University of Michigan. His primary research interests relate to the application of quantitative models to manufacturing and service systems, focusing on the design and development of supply chains and logistics. His e-mail address is segeie@rit.edu.

TOLGA BEKTAS is a Reader in Management Science at the University of Southampton and Director of M.S. Business Analytics and Management Sciences and M.S. Management Science and Finance at the Southampton Management School. He holds B.S., M.S., and Ph.D. degrees in Industrial Engineering with postdoctoral research experience at the University of Montreal. His research interests are in discrete optimisation with applications to vehicle routing, service network design, and freight transportation and logistics. He is an Editor of OR Insight and an Associate Editor of Computers and Operations Research. His publications have appeared in journals such as Transportation Research Part B, Transportation Science, Networks, European Journal of Operational Research, International Journal of Production Research, Omega, and the Journal of the Operational Research Society. His e-mail is t.bektas@soton.ac.uk.

JAVIER FAULIN is a Professor of Statistics and Operations Research at the Public University of Navarre (Pamplona, Spain). He holds a PhD in Economics, a MS in Operations Management, Logistics and Transportation and a MS in Applied Mathematics. His research interests include logistics, vehicle routing problems and simulation modeling and analysis. He is a member of INFORMS and EURO societies and an editorial board member of the International Journal of Applied Management Science and the International Journal of Operational Research and Information Systems. His e-mail address is javier.faulin@unavarra.es. 